Conclusions: Anxious or depressive patients showed higher disease activity, especially in measures with some subjectivity (such as TJC and PGA) but not regarding ESR or CRP and worse function and QoL. This fact must be taken into account when evaluating therapeutic efficacy.

Disclosure of Interest: None declared

DOI: 10.1136/annrheumdis-2017-eular.6345

\section{AB0330 RHEUMATOID FACTOR AND RO52KDA ANTIBODIES ARE INDEPENDENT PREDICTORS OF INSULIN RESISTANCE IN RHEUMATOID ARTHRITIS}

J. Aguilar-Arreola $^{1}$, F.D.J. Perez-Vazquez ${ }^{2}$, E. Gomez-Bañuelos ${ }^{2}$,

G.-I. Diaz-Rubio ${ }^{2}$, S. Duran-Barragan ${ }^{2}$, F.-I. Corona-Meraz ${ }^{2}$,

A. Saldaña-Millan ${ }^{2}$, R.-E. Navarro-Hernández ${ }^{2}, M$. Vázquez-Del Mercado ${ }^{2}$

${ }^{1}$ Servicio de Reumatología, División de Medicina Interna, Pnpc 004086

CONACyT, Hospital Civil, "Juan I. Menchaca"; ${ }^{2}$ Instituto de Invesgitación en

Reumatología y del Sistema Musculoesquelético, Universidad de Guadalajara,

Guadalajara, Mexico

Background: The rheumatoid factor (RF) and anti-citrullinated protein antibodies (ACPA) autoantibodies in rheumatoid arthritis (RA), have been used as diagnostic and prognostic tools [1]. However, this traditional perspective has changed toward a major role in RA pathogenesis. Several studies have demonstrated that FR and ACPA autoantibodies positivity beyond its level, might influence disease activity, bone erosions and development of comorbidities. Anti-Ro52kDa antibodies have also been associated with disease severity in RA and might influence the development of comorbidities such as insulin resistance (IR) in RA.

Objectives: To evaluate the association between RF, ACPA and anti-Ro52 kDa and IR in RA patients.

Methods: We included 83 RA patients classified according to ACR 1987 and ACR/EULAR 2010 criteria and 90 controls matched for age, gender and body mass index (BMI). Homeostasis Model Assessment-Insulin Resistance (HOMA-IR), anthropometric parameters and antibody positivity (RF, ACPA, Ro52 kDa) were evaluated. Multivariate regressión analysis was used to assess the contribution of autoantibodies, adiposity and disease activity to insulin resistance in RA.

Results: Patients positive for RF or anti-Ro52 kDa showed higher levels of basal insulin $(P=0.009, P=0.006)$ and HOMA-IR. DAS-28 ESR was correlated with basal insulin $(r=0.31, P=0.01)$ and HOMA-IR $(r=0.29, P=0.02)$. We also observed positive correlations between serum triglycerides $(r=0.47, P=0.01)$ and $H D L-c(r=-$ $0.38, P=0.02)$ and basal insulin. Multivariate analysis showed that Triglycerides, HDL-c, DAS-28, RF and anti-Ro52 kDa were independent predictors of basal insulin and HOMA-IR in patients with RA.

Conclusions: In RA, RF or anti-Ro52 $\mathrm{kDa}$ are independent predictors of IR. This phenomenon might be linked to the network of inflammation, adipokine secretion, since disease activity was also precitive of higher basal insulin. Both RF and anti Ro52 kDa, along with disease activity are independent predictors of IR in RA patients without comorbidities.

References:

[1] Watad A, Amital H: ACPAs Are Much More Than Diagnostic Autoantibodies. Rambam Maimonides Medical Journal 2016, 7(4).

[2] England BR, Thiele GM, Mikuls TR: Anticitrullinated protein antibodies: origin and role in the pathogenesis of rheumatoid arthritis. Current Opinion in Rheumatology 2016.

[3] Schuntermann MF: [References for evaluation scales in quality assurance in rehabilitation-1. I. Scales for determining adverse sequelae of illnesses-an introduction]. Die Rehabilitation 1995, 34(1):I-III.

[4] Matsudaira R, Tamura N, Sekiya F, Ogasawara M, Yamanaka K, Takasaki Y: Anti-Ro/SSA antibodies are an independent factor associated with an insufficient response to tumor necrosis factor inhibitors in patients with rheumatoid arthritis. Journal of Rheumatology 2011, 38(11):2346-2354.

Disclosure of Interest: None declared

DOI: 10.1136/annrheumdis-2017-eular.7003

\section{AB0331 BEHAVIOR OF THE VALUE OF RED CELL DISTRIBUTION WIDTH IN PATIENTS WITH RHEUMATOID ARTHRITIS IN TREATMENT WITH DAILY DOSE OF METOTREXATE}

J.E. Espinoza Martinez, M.U. Martinez MArtinez, C. Abud-Mendoza. Unidad de Reumatología y Osteoporosis, Hospital Central Dr. Ignacio Morones Prieto, San Luis Potosí, Mexico

Background: Recently the relationship between inflammatory biomarkers such as C-reactive protein (CRP) and erythrocyte sedimentation rate (ESR) has been found, with the increase in the percentage of red cell distribution width (RDW), events related to increase in cardiovascular risk in patients with rheumatoid arthritis (RA). RDW is a parameter that represents the heterogeneity of erythrocyte size and is calculated by an automatic blood analyzer, translates anisocytosis and in turn is related to atherosclerosis, is a predictor of mortality in patients with cardiovascular diseases such as acute myocardial infarction (AMI) and Congestive Heart Failure (CHF) plus it has the advantage of being very cheap. In patients with RA who receive treatment with methotrexate (MTX), particularly those with good therapeutic response with decreased disease activity, the values of ESR and CRP decrease.
Objectives: The aim of this study is to verify if there is a decrease, increase or neither change in the value of RDW in the patients receiving or not MTX comparing the value prior to the start of treatment and the last value measured during their therapy.

Methods: In this descriptive, non-experimental cross-sectional study, men and women older than 18 years of age with a diagnosis of rheumatoid arthritis according to ACR criteria (Aletaha et al., 2010) who were or not treated with methotrexate and other DMARDs. We excluded patients with less than two visits in this unit and the elimination criteria were patients who did not have baseline or last RDW test. The records of all patients included name, age, sex, date of diagnosis of RA, comorbidities, baseline and final laboratory exams during follow-up that included tests with RDW and medications.

Results: A total of 403 all with a diagnosis of RA and an average of 4.62 years of evolution, of which 51 they do not take methotrexate in daily dose and 352 receive treatment and only $4.2 \%$ suffered from a cardiovascular event. The comparison was made grouping the patients in whom they received and not treatment with methotrexate and correlated with the value of baseline and final RDW as shown in Table 1.

Table 1

\begin{tabular}{|c|c|c|c|c|}
\hline & \multicolumn{4}{|c|}{ Methotrexate } \\
\hline & \multicolumn{2}{|c|}{ Receive } & \multicolumn{2}{|c|}{ Do not Receive } \\
\hline & Baseline RDW & Final RDW & Baseline RDW & Final RDW \\
\hline Minimum & 10.70 & 10.40 & 11.10 & 12.20 \\
\hline Median & 15.70 & 15.70 & 15.85 & 15.35 \\
\hline Mean & 15.97 & 16.01 & 15.97 & 15.93 \\
\hline Maximum & 32.60 & 26.60 & 27.30 & 21.80 \\
\hline
\end{tabular}

The results shown in Table 1, do not appear to reveal a significant change in RDW values between the different subgroups; in the figures we compared the median of RDW for each group of patients who take methotrexate from all patients included in this study.
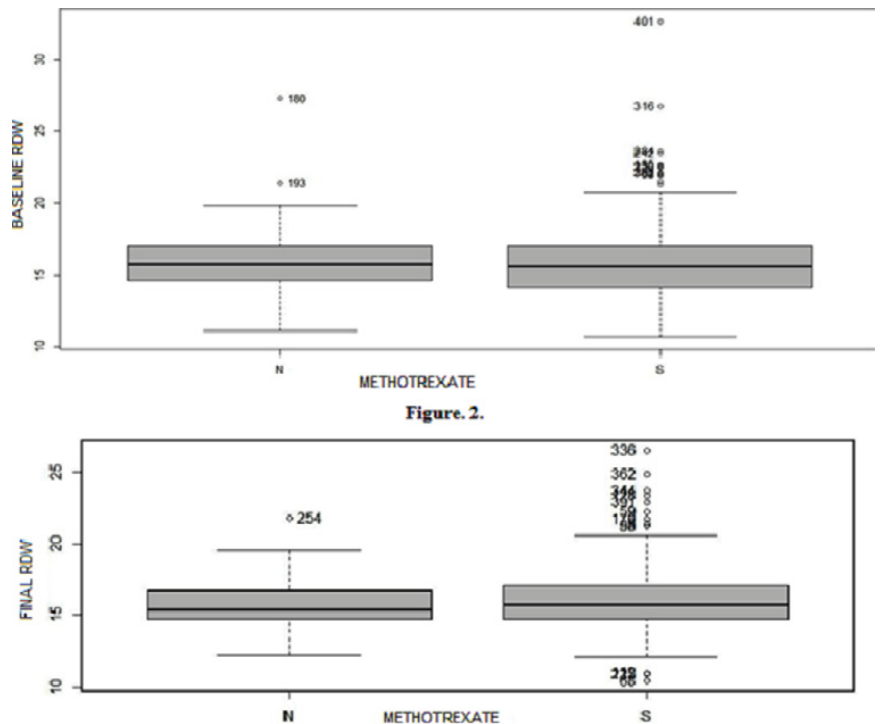

Conclusions: The value of RDW does not appear to significantly change its value when taking methotrexate at a daily dose in RA patients. The value of RDW may have weight in the assessment of the risk of suffering a cardiovascular event in patients with rheumatoid arthritis.

References:

[1] Hassan, S. et al. (2015). Red Cell Distribution Width: a measure of cardiovascular risk in rheumathoid arthtritis patiens? Clin Rheumatol.

[2] Woong Soo, L. et al. (2010). Relation between red cell distribution width and inflammatory biomarkers in rheumatoid arthritis. Seoul, Republic of Korea.

[3] Aletaha, et al. (2010). 2010 Rheumatoid arthritis classification criteria, Arthritis \& Rheumatism.

Disclosure of Interest: None declared

DOI: 10.1136/annrheumdis-2017-eular.3896

\section{AB0332 DOES A COMMUNITY INTERFACE RHEUMATOID ARTHRITIS ANNUAL REVIEW IMPROVE PATIENT CARE?}

J. Mcdonald, R. Haigh, D. Murphy. Rheumatology, Royal Devon and Exeter Hospital, Exeter, United Kingdom

Background: Patients with rheumatoid arthritis are known to have a long term disability and increased risk of extra-articular comorbidities. EULAR guidelines suggest annual review of cardiovascular risk in patients with rheumatoid arthritis [1] whilst UK national (NICE) guidelines suggest a more holistic annual review to look at the impact of the disease on quality of life as well as co-morbidities [2] 
Objectives: Our aim was to look at the annual reviews currently taking place in primary care to see how frequently patient co-morbidities were assessed and documented. We then implemented a formal community rheumatology interface review to assess whether this improved patient care.

Methods: A large primary care practice (16,000 patients) was offered a community rheumatology interface review by a secondary care clinician. A search was undertaken for patients with rheumatoid arthritis who had attended for an annual primary care review between December 2015-2016. Of these 30 reviews were selected and we assessed how frequently the following were recorded; DAS28, HAQ score, FRAX score, Q-Risk2 (CV risk assessment tool) and screening for depression. Once we had analysed these results we implemented a community rheumatology interface review and assessed compliance with the above outcomes had improved compared to standard primary care management. Results: In patients assessed prior to implementation of interface review, we found that a DAS28 score was recorded in $0 \%, \mathrm{HAQ}$ score in 0\%, FRAX in $13 \%$, Q-Risk2 in 10\% and depression screening in $23 \%$.

In comparison, patients assessed by a community rheumatology interface clinician recorded DAS28 in 100\%, HAQ score in 100\%, FRAX in 100\%, Q-Risk2 in $100 \%$ and depression screening was recorded in $100 \%$. Based on improved interface review 7 patients $(23 \%)$ were sent for DEXA scanning or started on a bisphosphonate, we discussed cardiovascular risk and starting a statin in 8 patients $(26 \%)$ and 7 patients $(23 \%)$ required follow-up for mental health. Of the 30 patients in this cohort all patients reported full adherence to their anti-rheumatic regime.

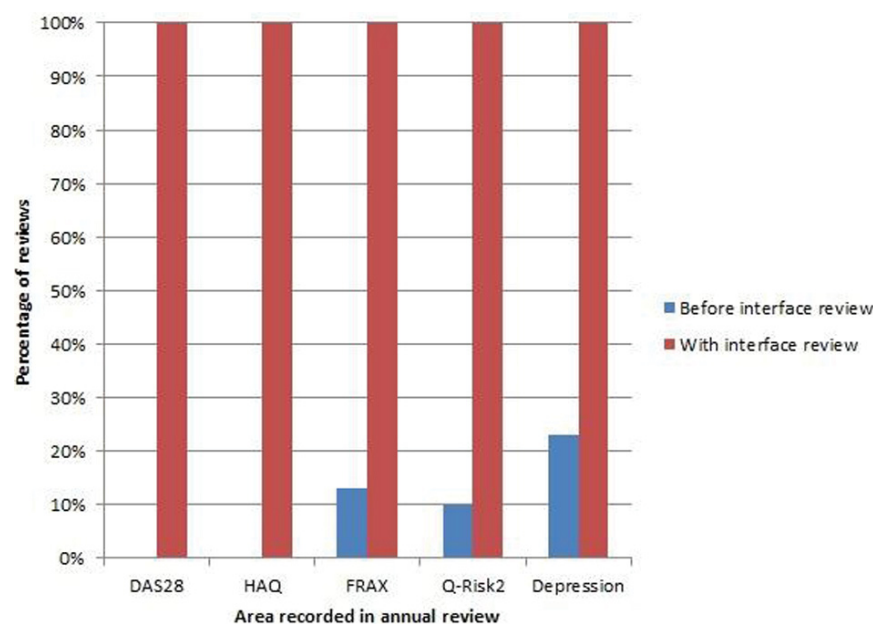

Conclusions: 1. An annual review with a rheumatology interface practitioner is of benefit in holistic patient care and improved compliance with all domains of the annual review.

2. Management of metabolic bone, cardiovascular and mental health issues was improved, according to current national guidelines.

References:

[1] Peters $M$ et al. EULAR evidence-based recommendations for cardiovascular risk management in patients with rheumatoid arthritis and other forms of inflammatory arthritis. Ann Rheum Dis 2010 69:325-331.

[2] National Institute for Health and Clinical Excellence (NICE) (2009). Rheumatoid Arthritis in Adults: Management. Clinical Guideline 79. London: NICE.

Disclosure of Interest: None declared

DOI: 10.1136/annrheumdis-2017-eular.4804

\section{AB0333 USE OF BIOLOGICAL DMARDS, BUT NOT OF CONVENTIONAL SYNTHETIC DMARDS OR STEROIDS, MAY BE RELEVANT TO INCREASED ENERGY INTAKE IN PATIENTS WITH RHEUMATOID ARTHRITIS}

K. Masuko ${ }^{1,2}$, Y. Mizukami ${ }^{3}$, S. Tohma ${ }^{4}$, T. Matsui ${ }^{5} .{ }^{1}$ Clinical Research Center for Medicine, International University of Health and Welfare; ${ }^{2}$ Health Evaluation and Promotion Center, Sanno Medical Center, Tokyo; ${ }^{3}$ Graduate School of Nutritional Science, Sagami Women's University; ${ }^{4}$ Clinical Research Center for Allergy and Rheumatology, National Hospital Organization Sagamihara National Hospital, Kanagawa; ${ }^{5}$ Graduate School of Medical and Dental Sciences, Tokyo Medical and Dental University, Tokyo, Japan

Background: Dietary and nutritional factors may modulate the onset and/or outcome and comorbidities including cardiovascular diseases in rheumatoid arthritis (RA)[1]. For instance, a potential contribution of sodium intake and the occurrence of autoimmunity has been suggested, however the role of sodium in disease activity of RA is unclear.

Objectives: To investigate the potential relationship between dietary intake and current medications and the disease activity of RA.

Methods: Patients with RA in outpatient clinic of National Hospital Organization, Sagamihara National Hospital $(n=89, M / F=18 / 71$, age $65.0 \pm 12.8$ yo, mean disease duration $16.8 \pm 13.2 \mathrm{y}$ ) were enrolled to the study. A questionnaire regarding dietary habit and the brief-type self-administered diet history questionnaire (BDHQ) were used to assess the relationship between clinical activity, status of medication and intake of each foodstuff and nutritional factors. The results were analyzed using IBM SPSS software using Pearson's correlation test and Levene test.

Results: The number of current medication was found to weakly correlate with DAS-28 $(r=0.386)$, and negatively with intake of ethanol $(r=-0.277)$. When users of biological disease modulating anti-rheumatic drugs (bDMARDs) were compared with non-users, the biologics-taking patients tended to have higher intake of total energy and carbohydrates than non-users. Use of oral corticosteroids, nor other anti-rheumatic DMARDs, did not significantly impact the total energy or carbo intake in RA patients. Sodium intake did not correlate with the disease status or the number of medications.

Conclusions: Although salt intake has been suggested to evoke autoimmunity, the amount of dairy intake of salt does not significantly modulate the current disease activity of RA. Use of biologics, but not of corticosteroids, may lead to increased energy and carbohydrate intake in RA patients.

\section{References:}

[1] Mizukami Y, et al. Distinct Patterns of Dietary Intake in Different Functional Classes of Patients with Rheumatoid Arthritis. Topics in Clinical Nutrition (in press).

Disclosure of Interest: None declared

DOI: 10.1136/annrheumdis-2017-eular.4603

\section{AB0334 KNEE FUNCTION AFTER TOTAL KNEE ARTHROPLASTY IS INFLUENCED BY DISEASE ACTIVITY IN PATIENTS WITH RHEUMATOID ARTHRITIS}

K. Harigane ${ }^{1,2}$, K. Kumagai $^{2}$, Y. Mochida ${ }^{1}$, K. Ishii ${ }^{1}$, Y. Miyamae ${ }^{1}$, H. Choe ${ }^{2}$, A. Nagaoka ${ }^{2}$, Y. Inaba ${ }^{2}$, N. Mitsugi ${ }^{3}$, T. Saito ${ }^{2} .{ }^{1}$ Center for Rheumatic Diseases, Yokohama City University Medical Center; ${ }^{2}$ Department of Orthopaedic Surgery, Yokohama City University; ${ }^{3}$ Department of Orthopaedic Surgery, Yokohama City University Medical Center, Yokohama, Japan

Background: Several studies reported that development of phaemacological treatment for rheumatoid arthritis (RA) contributed to decreased number of orthopaedic surgery. [1-3] Surgical treatment is, however, still required in many cases, and the impact of orthopaedic surgery on disease activity remain unclear. Objectives: The aims of current study was to evaluate the effect of total knee arthroplasty (TKA) with capsulosynovectomy on changes of disease activity and knee function after TKA in patients with RA.

Methods: Seventy-seven serial patients with RA (61 female and 16 male) who underwent primary TKA with more than one year of follow-up were retrospectively reviewed to assess postoperative disease activity and knee function. The mean age at the time of surgery was 68.3 years old. The disease activity of RA was measured using Disease Activity Score in 28 Joints (DAS28). Clinical outcome was measured by treatment score for RA knee of the Japanese Orthopaedic Association (JOA) score. To evaluate the effects of disease activity on knee function, outcomes at before and one year after surgery were separately investigated following two groups; patients who had remission or low disease activity in DAS28-CRP (good controlled group), and patients who had moderate or high disease activity (poor controlled group) one year after surgery.

Results: The disease activity of RA was significantly decreased in DAS28-CRP one year after surgery. ( 3.9 vs. $2.7, p<0.01)$ Postoperative knee function was significantly improved in JOA scores one year after surgery. (48.9 vs. 86.0, $p<0.01)$ As for differences of knee function between good and poor controlled group, the mean JOA score in good controlled group was significantly better than in poor controlled group. (90.4 vs. 82.1, $\mathrm{p}<0.01$ ) Postoperative knee function was negatively correlated with $R A$ disease activity. $\left(R^{2}=0.21, p<0.01\right)$

Conclusions: TKA with capsulosynovectomy improves both knee function and disease activity in patients with RA. Based on the results, knee function after TKA is influenced with disease activity.

References:

[1] Dusad A et al. Impact of Total Knee Arthroplasty as Assessed Using PatientReported Pain and Health-Related Quality of Life Indices: Rheumatoid Arthritis Versus Osteoarthritis. Arthritis Rheumatol. 2015; 67: 2503-11.

[2] Yano K et al. Effect of total knee arthroplasty on disease activity in patients with established rheumatoid arthritis: 3-year follow-up results of combined medical therapy and surgical intervention. Mod Rheumatol. 2010; 20: 452-7.

[3] Oh $\mathrm{K}$ et al. Effects of surgical intervention on disease activity of rheumatoid arthritis: cases of surgery for rheumatoid arthritis of the lower limbs treated with biologics. Mod Rheumatol. 2014; 24: 606-11.

Disclosure of Interest: None declared

DOI: 10.1136/annrheumdis-2017-eular.3029 